\title{
RELATIVE NAVIGATION FOR THE TANDEM-X MISSION AND EVALUATION WITH DEM CALIBRATION RESULTS
}

\author{
Martin Wermuth \\ German Space Operations Center (DLR/GSOC), Oberpfaffenhofen 82234 Wessling, Germany \\ +498153282155, martin.wermuth@dlr.de \\ Oliver Montenbruck \\ German Space Operations Center (DLR/GSOC), Oberpfaffenhofen 82234 Wessling, Germany \\ +498153281195, oliver.montenbruck@dlr.de
}

\author{
Anna Wendleder \\ German Remote Sensing Data Center (DLR/DFD), Oberpfaffenhofen 82234 Wessling, Germany +49 8153 \\ 283439, anna.wendleder@dlr.de
}

\begin{abstract}
The TanDEM-X mission is designed to acquire a global digital elevation model (DEM) with an unprecedented accuracy of $2 \mathrm{~m}$ at a grid size of $12 \mathrm{~m} \times 12 \mathrm{~m}$. This is done by bi-static synthetic aperture radar (SAR) data takes from the TSX and TDX satellites. In order to achieve the required accuracy, the baseline vector between the two spacecraft has to be determined with an accuracy of $1 \mathrm{~mm}$ (ID-RMS). The baseline is computed in a relative navigation using the measurements of the on-board GPS receivers combined with dynamic models. The accuracy of the baseline will be evaluated with dedicated calibration data takes over well known areas. Systematic errors can be detected and corrected for.
\end{abstract}

Keywords: TanDEM-X, baseline determination, relative navigation, digital elevation model, GPS

\section{Introduction}

\subsection{The TanDEM-X mission}

The TanDEM-X mission (TerraSAR-X add-on for Digital Elevation Measurements) is a German dual satellite mission with the task to acquire a high quality digital elevation model of the whole Earth surface. It is the first mission to operate two satellites in close formation and acquire height information by bi-static radar data-takes.

The mission consists of two almost identical spacecraft: TSX which was launched in June 2007 with a Russian Dnepr rocket in Baikonur, and TDX which followed three years later in June 2010 with the same launcher. Both satellites have a hexagonal shape with a length of about $5 \mathrm{~m}$, a diameter of $2.4 \mathrm{~m}$ and a weight of more than 1200 $\mathrm{kg}$. They are operated in a sun-synchronous orbit at an orbit height of $515 \mathrm{~km}$ and an inclination of $97.44^{\circ}$.

The main payload of both satellites is a X-band synthetic aperture radar (SAR) antenna. Each of the two spacecraft can acquire data takes with its SAR instrument on its own as part of the TerraSAR-X mission. For digital elevation measurements the satellites are operated in the bi-static mode. This means that only one satellite is sending radar pulses, but both satellites are receiving the signals reflected by the Earth's surface (see Fig. 1 or refer to Krieger et al., 2007). The instrument is pointing towards the right with an angle to the nadir direction of $33^{\circ}$ (seen in flight direction). If the area of interest of a data take is to the left, the whole satellite can be rotated to perform a so-called left-looking data take.

In addition, the satellites are equipped with the TOR instrument (Tracking, Occultations and Ranging) provided by the GeoForschungsZentrum Potsdam (GZF). It consists of the Integrated GPS and Occultations Receiver (IGOR) and a laser retro reflector. The IGOR is a high-grade geodetic dual-frequency GPS receiver which has 12 channels allocated for the tracking of GPS satellites for navigation needs (and four additional channels for occultation observations). This allows for a precise determination of the single satellites orbits and the baseline vector between the two satellites.

In order to enable the bi-static DEM acquisition, the satellites are operated in a close helix formation with a distance of less than $500 \mathrm{~m}$ (see Kahle and Schlepp, 2010). The TSX satellite is considered the reference 
satellite, which follows a pre-defined reference orbit with a repeat cycle of 11 days and 167 revolutions. The maximum deviation from that reference orbit is $250 \mathrm{~m}$. Orbit control maneuvers with the hydrazine thrusters are currently executed about once per week. The frequency could increase with increasing solar flux. The TDX satellite is controlled relative to the TSX satellite. It replicates all hydrazine maneuvers and additionally keeps the formation daily with a pair of cold gas maneuvers.



Figure 1. The TanDEM-X mission (courtesy: DLR)

\subsection{Requirements on baseline accuracy}

The goal of the TanDEM-X mission is to create a global digital elevation model (DEM) with the unprecedented accuracy of $2 \mathrm{~m}$ over a horizontal grid of $12 \mathrm{~m}$ x $12 \mathrm{~m}$. Therefore the baseline error has to be kept minimal, as a baseline error will not only induce a height error and tilt in the DEM but also a vertical displacement. The height error $\Delta \mathrm{h}$ which results from a baseline error in line of sight $\Delta B_{\mathrm{LOS}}$ can be computed by:

$$
\Delta h=\frac{h_{a m b}}{\lambda} \Delta B_{L O S},
$$

where $\lambda$ is the wavelength of the SAR signal and $h_{\mathrm{amb}}$, the height of ambiguity, is a factor depending on the satellite geometry (see Krieger et al., 2010):

$$
h_{a m b}=\frac{\lambda r_{0} \sin \theta}{B_{\perp}} .
$$

$B_{\perp}$ is the so-called effective baseline i.e. the vector between the satellites projected on the line of sight, $r_{0}$ is the slant range and $\theta$ the incidence angle of the data take. At a wavelength of $\lambda=3.1 \mathrm{~cm}$ (X-Band) and a typical $h_{\mathrm{amb}}$ $=30 \mathrm{~m}$, a baseline error in line of sight of $1 \mathrm{~mm}$ would translate to a DEM height error of $1 \mathrm{~m}$.

Furthermore, a baseline error will cause a tilt of the DEM scene, which can be estimated by:

$$
\varphi_{\text {tilt }}=\frac{\Delta B_{L O S}}{B_{\perp}} .
$$


Assuming a typical $B_{\perp}$ of $300 \mathrm{~m}$, a baseline error in line of sight of $1 \mathrm{~mm}$ would cause a tilt of $0.0002^{\circ}$. This would cause a height variation of 0.1 m over a DEM scene with a swath width of $30 \mathrm{~km}$.

When a raw DEM containing a height error and tilt is geocoded using overlapping raw DEMs and DEMs from former missions (like the Shuttle Radar Topography Mission SRTM), the errors cause a displacement of the DEM (see Fig. 2). The displacement can be estimated by:

$$
\Delta x=\frac{1}{\tan \theta} \Delta h,
$$

where $\theta$ is the incidence angle (the angle from which the line of sight deviates from the nadir direction). This means, that e.g. during a near range data take under an incidence angle of $27^{\circ}$, the displacement $\Delta x$ is twice the height error $\Delta h$. Hence already a baseline error of $3 \mathrm{~mm}$ would cause a height error of $3 \mathrm{~m}$, which could again cause a displacement of $6 \mathrm{~m}$. With a DEM pixel size of $12 \mathrm{~m}$ this shift of more than half a pixel would cause the wrong pixels in two raw DEMs to be matched. Therefore, a requirement for the baseline accuracy of $1 \mathrm{~mm}$ RMS in $1 \mathrm{D}$ was raised.

\subsection{Filter for relative navigation of spacecraft (FRNS)}

The Filter for Relative Navigation of Spacecraft (FRNS) is part of the GPS High precision orbit determination Software Tools (GHOST), which were developed at the German Space Operations Center (DLR/GSOC) in cooperation with TU Delft. It is designed to perform a relative orbit determination between two LEO spacecrafts. This is done using an extended Kalman filter described in Kroes (2006). The concept is to achieve a higher accuracy for the relative orbit between two spacecrafts by making use of differenced GPS observations, than by simply differencing two independent orbit determination results. FRNS requires a continuous reference orbit for both spacecrafts, such as the science orbit product, which is computed on a routine basis by GSOC. It then keeps the orbit of one spacecraft fixed, determines the relative orbit between the two spacecrafts and adds it to the positions of the first spacecraft. As result a trajectory file containing the orbits of both spacecrafts is obtained. The tool is able to process both single and dual-frequency observations.

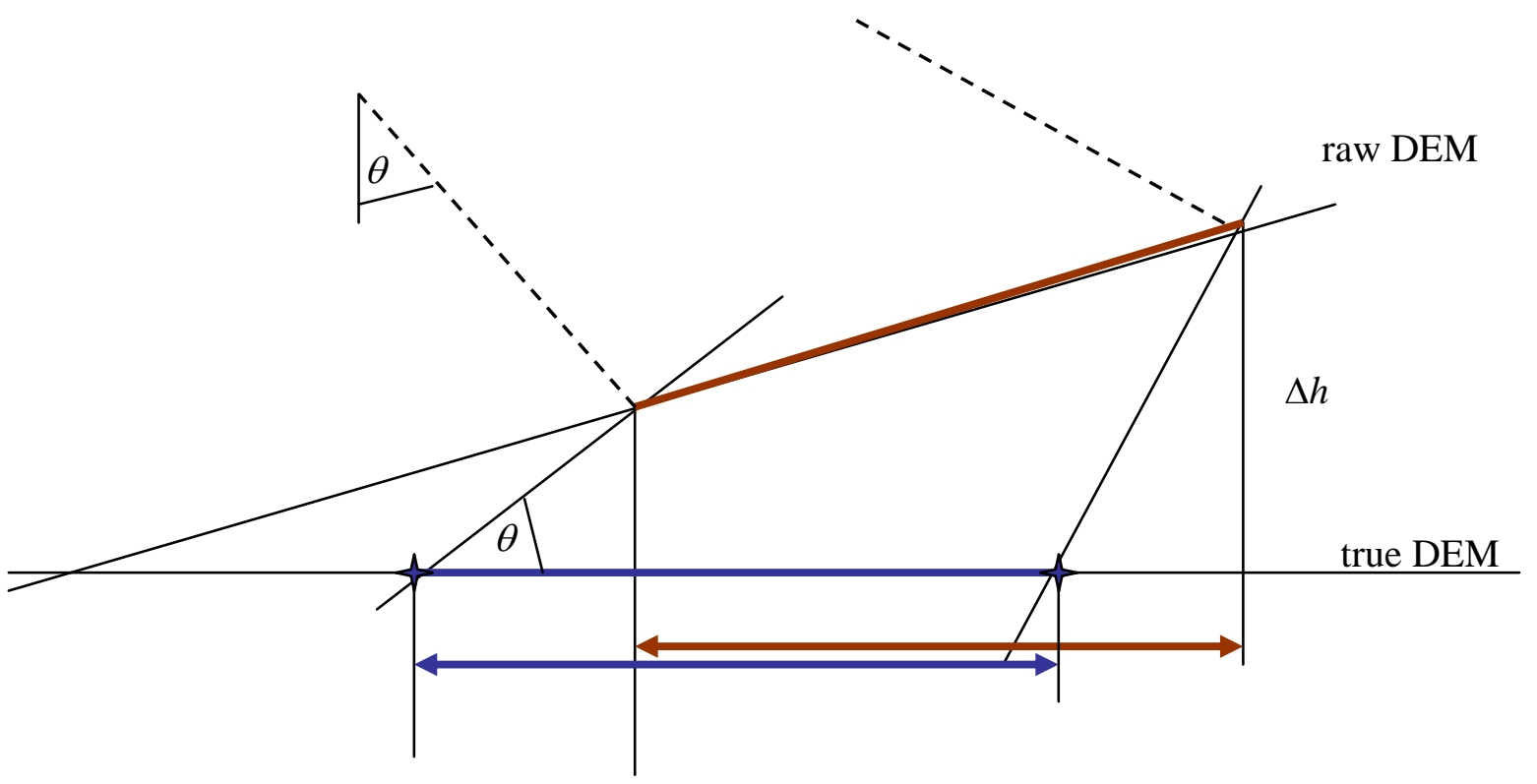

Figure 2. Displacement of a raw DEM due to height error and tilt

\subsection{Experience from the GRACE mission}

The FRNS tool was developed using data from the GRACE mission. In contrast to TanDEM-X, GRACE consists of two spacecraft, which follow each other on a similar orbit with about $200 \mathrm{~km}$ distance. The distance between the two spacecraft is measured by a K-band link, which is considered to be at least one order of magnitude more 
accurate than GPS observations. Hence the K-band observations can be used to assess the accuracy of the relative navigation results - with the limitation, that the K-band observations only reflect the along-track component, and contain an unknown bias. The differences between a GRACE relative navigation solution and K-band observations are shown in Fig 3.

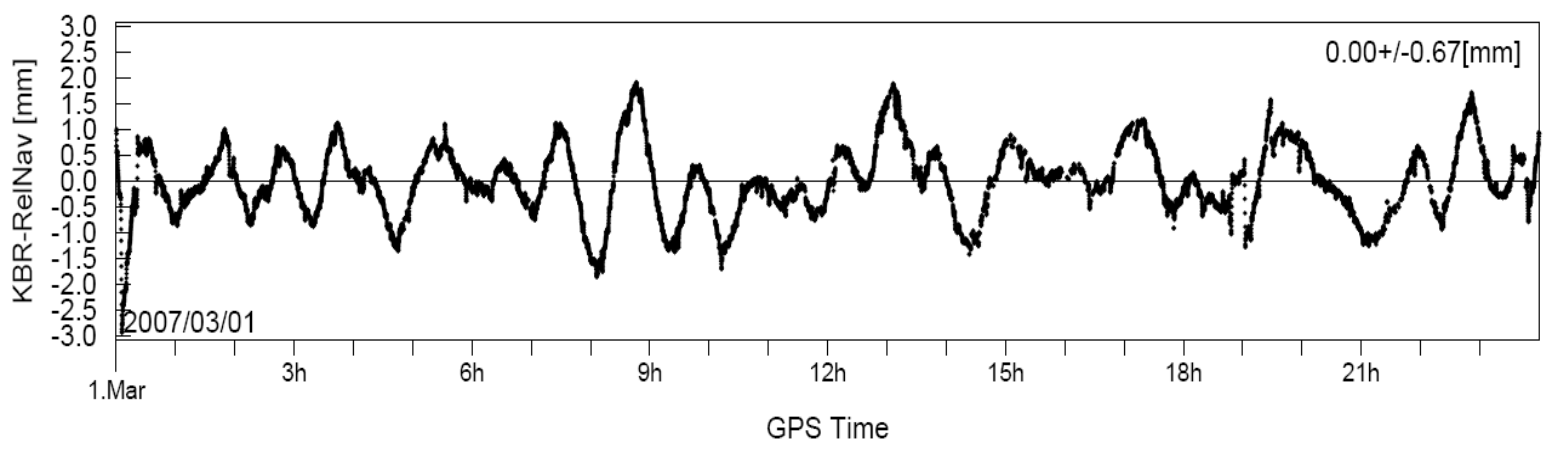

Figure 3. Comparison of GRACE relative navigation solution with K-band observations

As the IGOR receiver is a follow-on model of the BlackJack receiver used on GRACE it can be expected, that for TanDEM-X the same level of accuracy can be achieved in the baseline determination. Due to the smaller distance between the TSX and TDX satellites, the influence of the ionosphere on the baseline quality should be minimal.

Nevertheless comparison of GRACE baseline determination results with results determined by the Astronomical Institute of the University Berne (AIUB) using an independent software show that inconsistencies exist between the two solutions (see Jäggi et al., 2009). The differences show a systematic bias of up to $2 \mathrm{~mm}$ per component and standard deviations of up to $1.5 \mathrm{~mm}$ (largest deviations occur in the component normal to the orbit plane). Assuming that both solutions are independent, the 1D-sigma requirement is fulfilled, but the biases have to be detected and corrected to reach a 1D-RMS requirement of $1 \mathrm{~mm}$. As there is no K-band ranging on the TanDEM$\mathrm{X}$ mission which could serve as external tool to assess the quality of the baseline determination, it has been decided to use so-called baseline calibration data takes for the evaluation of a possible baseline bias.

\section{Baseline processing}

\subsection{Data screening and availability}

For the baseline determination using the FRNS software it is important to have common GPS observations on both satellites. At the beginning of the commissioning phase an average of 8.2 GPS satellites was tracked on TSX and 8.7 on TDX, but only 7.7 satellites were in average tracked simultaneously on both TSX and TDX. As the newest GPS satellite (G25) had no valid phase center calibration at that time it was not used for baseline determination and only an average of 7.5 common satellites were available. Nevertheless this is still more, than the 6.5 common observations which are typically available for GRACE baseline determination.

An investigation by the provider of the IGOR receiver (GFZ), revealed that observations were lost on TSX while occultation observations (a secondary mission objective) were enabled. The loss of observations occurred due to the fact, that one GPS satellite was selected as clocksat and the corresponding observations were simply encoded differently in the telemetry data. A modification in the data pre-processing made the observations available again. The GPS observation data was reprocessed from DOY 200/2010 on, making the observations of the clocksat available again. This led to an increase of the simultaneously tracked satellites on TSX by 0.4 to 8.6 (this means, that a clocksat was assigned $40 \%$ of the time). As a phase center calibration for the G25 satellite was now available, it was included in the baseline processing. This finally led to an average of 8.2 commonly observed satellites.

As mentioned above, the FRNS software determines the precise baseline with a reduced dynamic approach. This means that a numerically integrated trajectory based on dynamic models is fit to the GPS observations. The software furthermore provides an alternative kinematic approach, which is independent of dynamic models (and their possible deficiencies) at the cost of a larger noise in the estimated position. The results of the kinematic baseline determination were used as a first internal validation of the reduced dynamic baseline determination. The validation showed a systematic difference of about $7 \mathrm{~mm}$ in the radial component between reduced dynamic and kinematic baseline solution (see Fig. 4). While the large standard deviation of more than $10 \mathrm{~mm}$ can be explained by the noise of the kinematic solution, this systematic difference was unexpected, as the experience 
from the GRACE mission showed that systematic biases between different software approaches should not be larger than $2 \mathrm{~mm}$. An investigation by the satellite manufacturer Astrium revealed that in fact the center of mass coordinates of the TDX satellite were off by $7 \mathrm{~mm}$. After a reprocessing with the corrected coordinates, the systematic bias between reduced dynamic and kinematic processing vanished.

\subsection{Routine processing}

The TanDEM-X baseline is computed at GSOC automated on a routine basis in daily arcs with a latency of seven days. The latency was chosen to ensure, that all necessary auxiliary data is available and consolidated. The daily arcs span over 30 hours - starting at 21:00 h of the previous day till 03:00 h on the following day - to create a 6 hours overlap between consecutive baseline products. These overlap periods serve as internal quality check. If the difference between two products exceeds a certain threshold, the automatic product delivery is stopped and the baseline product has to be examined manually by an operator.
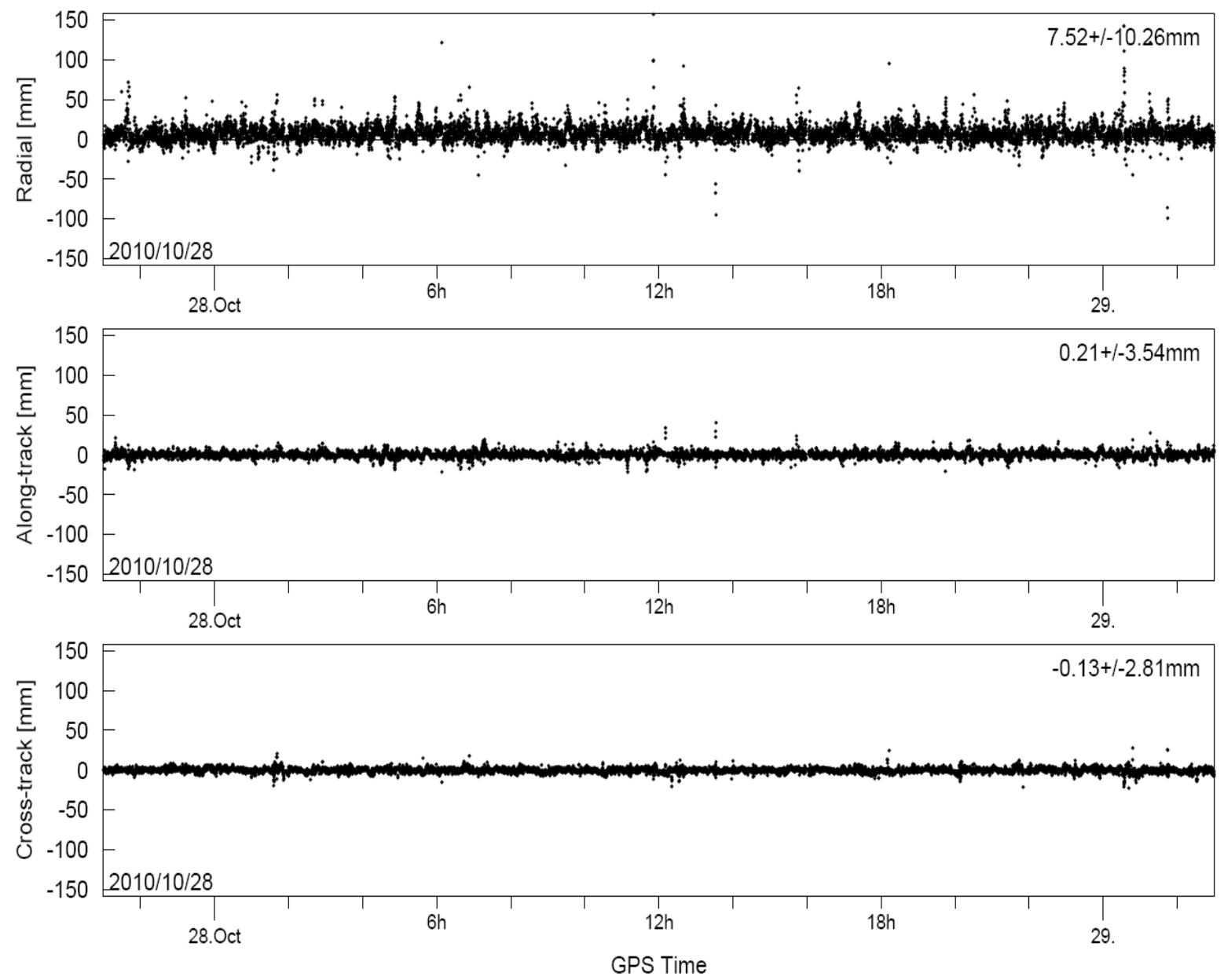

Figure 4. Difference of reduced dynamic and kinematic baseline solutions on DOY 301/2010

The GPS processing is based on the rapid GPS ephemerides provided by the Center for Orbit Determination in Europe (CODE). As further auxiliary data, the satellites' attitude information is used as input data. For dynamic modeling the GRACE gravity field GGM01 and the ocean tide model TOPEX 3.0 are employed. Due to regular orbit and formation control maneuvers, the fuel tanks of the satellites will empty causing a shift of the center of mass of up to $10 \mathrm{~cm}$ over the live-time of the satellites. This requires a regular update of the center of mass coordinates in relation to the fuel consumption to ensure the high accuracy of the baseline determination. For further details on the processing concept refer to Montenbruck (2010).

In addition to overlap comparisons between two baseline arcs, FRNS provides further tools to asses the quality of the baseline solution, which are as well included as pass criteria in the automated processing. On the one hand GPS measurement residuals are computed. If they exceed a certain threshold, the automated processing is stopped as well. On the other hand, the short distance between the two satellites opens the possibility of a singlefrequency baseline determination. The differences in Ionospheric delays are so small, that the ambiguity 
resolution works much more stable for a single frequency solution than for the dual frequency solution. Although the single frequency solution is less accurate, it can be used to detect errors in the dual frequency version.

\subsection{Results}

An overlap plot in radial-tangential-normal coordinates is shown for DOY 300/301 2010 in Fig. 5. Since the start of the close formation, the 3D-RMS difference between two consecutive arcs is $0.2 \mathrm{~mm}$. Nevertheless it should be stated, that this number does not reflect the actual accuracy of the baseline products. It rather shows the accuracy of repeatability of the results and seems to be too optimistic as parameter for the overall accuracy.
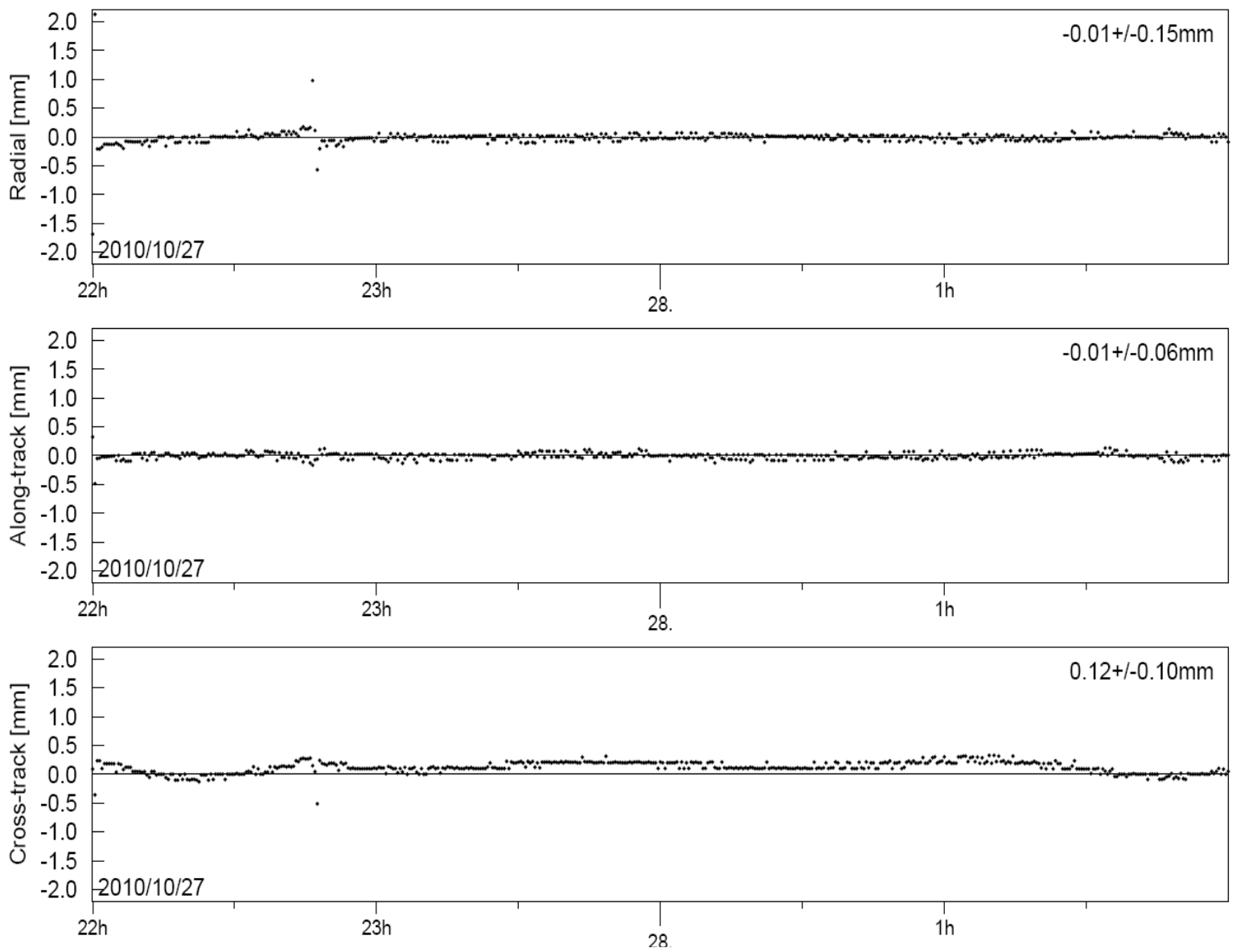

Figure 5. Overlap comparison between the solution of DOY 300/2010 and DOY 301/2010

As a second internal quality criterion, the GPS measurement residuals are analyzed. After removal of outliers, the pseudorange observations residuals have an RMS of $21 \mathrm{~cm}$ on both frequencies. The L1 carrier phase residuals have an RMS of $1.0 \mathrm{~mm}$ and the L2 residuals have an RMS of $0.7 \mathrm{~mm}$. A residual plot for DOY 301 is shown in Fig. 6. The carrier phase residuals achieved with data from the GRACE mission $(0.9 \mathrm{~mm}$ for L1 and $0.7 \mathrm{~mm}$ for L2) are slightly better, than those of TanDEM-X.

A plot of the differences between dual-frequency and single-frequency solution is shown for DOY 301 in Fig. 7. A statistic of the differences is given in Tab. 1 distinguishing between a wide formation with a distance of $20 \mathrm{~km}$ between the satellites (flown at the beginning of the mission) and the close formation with a distance of less than $500 \mathrm{~m}$. It can be seen, that the two solutions show a good consistency especially in the close formation. This reflects an improvement of the single-frequency solution with a smaller distance between the two spacecraft due to a decreasing influence of the differential ionosphere.

Table 1. Comparison between dual- and single-frequency solutions

\begin{tabular}{|l|c|c|c|c|}
\hline & Radial [mm] & Tangential [mm] & Normal [mm] & 3D-RMS \\
\hline wide formation & $0.1+/-1.0$ & $2.1+/-1.6$ & $1.5+/-0.9$ & 3.5 \\
\hline close formation & $0.0+/-0.7$ & $1.0+/-0.8$ & $1.2+/-0.7$ & 2.1 \\
\hline
\end{tabular}





Figure 6. GPS residuals for DOY 301/2010

As can be seen in Fig. 7, the two solutions show large deviations of up to $10 \mathrm{~mm}$ (with one outlier of $30 \mathrm{~mm}$ ) during maneuvers. There are two pairs of cold gas maneuvers, which are typical for formation control during the DEM acquisition. The first pair occurs between $22: 00 \mathrm{~h}$ and 24:00 $\mathrm{h}$ on 2010/10/27 and the second between 20:00 $\mathrm{h}$ and 22:00 $\mathrm{h}$ on 2010/10/28. The largest deviations affect only a couple of samples (30s sampling rate used for this plot), but the disturbances cover the period of half an hour before the first maneuver till half an hour after the second maneuver of each pair. It should be noted, that the single-frequency solution is to be considered weaker than the dual-frequency version. Hence it is not necessarily true, that the errors of the dual-frequency product amount to $10 \mathrm{~mm}$ during maneuvers. 

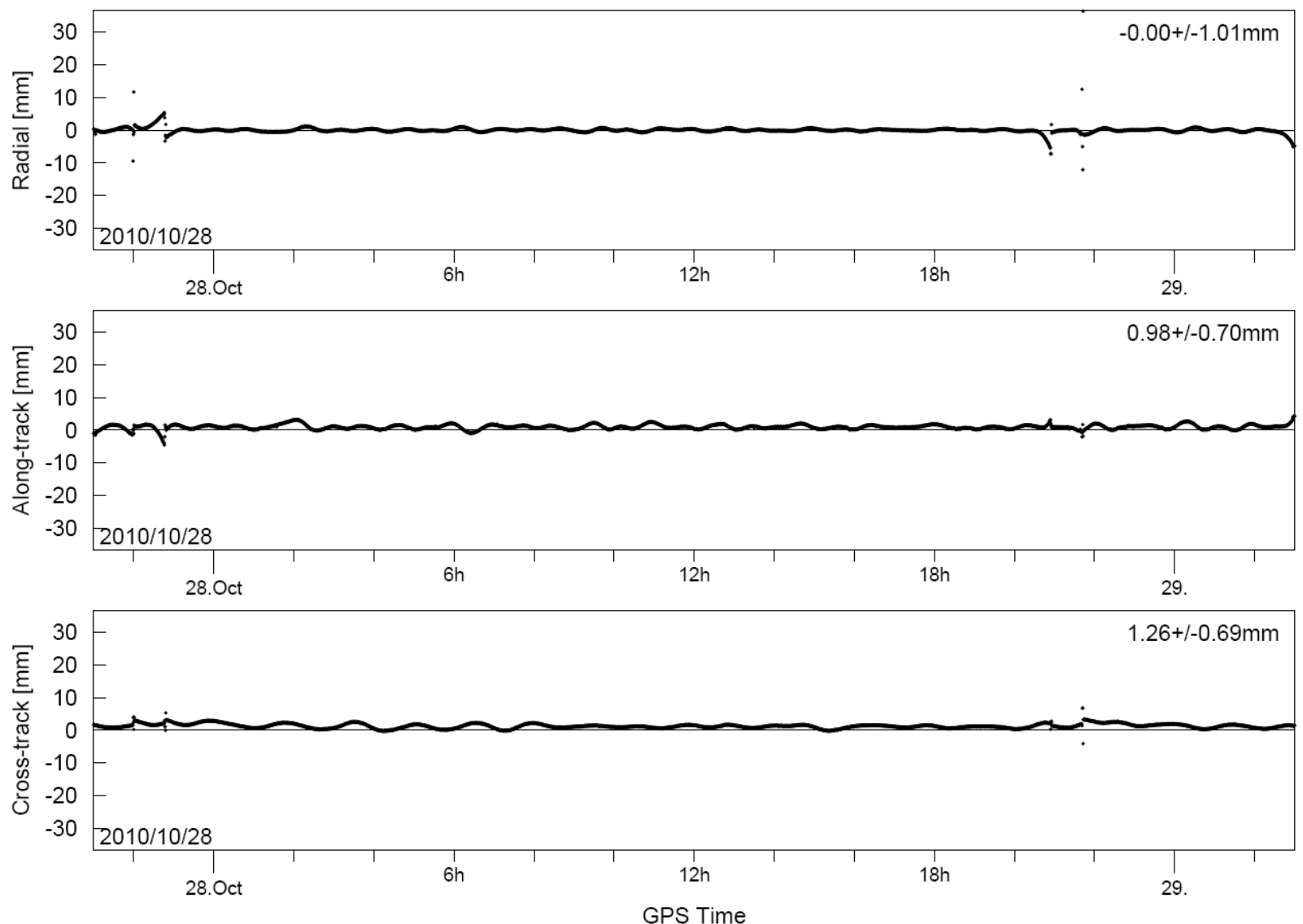

Figure 7. Comparison between dual- and single-frequency solution for DOY 301/2010

\section{Validation with baseline calibration data takes}

As mentioned above, the experience obtained with baseline determination of the GRACE mission hints that constant biases in the order of up to $2 \mathrm{~mm}$ remain in the baseline solution. Hence it was decided to perform regular baseline calibration data takes to gather information about a possible baseline bias. These calibration data takes are acquired over areas with a well known DEM from previous missions (like SRTM) and preferably flat terrain. Since the close formation was reached and bi-static operations have begun, almost 700 bi-static baseline calibration data takes have been acquired between October and December 2010 and evaluated.

For the evaluation, the raw DEMs created by the calibration data takes have been compared to SRTM DEMs, which were corrected by ICESat data. As "Eq. 1" shows the relation between height error and baseline error, this relation can be inverted to derive the baseline error in line of sight from the height difference between the TanDEM-X raw DEM and the geocoded SRTM DEM:

$$
\Delta B_{L O S}=\frac{\Delta h \cdot \lambda}{h_{a m b}} .
$$

As systematic baseline errors are assumed to be constant in the local R-T-N system (radial - tangential - normal), the calibration data takes have been taken under three different incident angles $\left(\square=31^{\circ}, 40^{\circ}, 47^{\circ}\right)$ in order to separate radial errors from errors in normal direction. Unfortunately no conclusions about the error in along-track (resp. tangential) direction can be drawn, as the line of sight of all data takes is perpendicular to the flight direction.

Figure 8 and Fig. 9 show the example of a baseline calibration data take over Australia. The left image of Fig. 8 shows the raw DEM derived from the data take. The right image shows the corresponding scene from the SRTM database and Fig. 9 the height difference between the two DEMs. The average height difference between those two DEMs is $-4.730 \mathrm{~m}$ with a standard deviation of $3.360 \mathrm{~m}$. The height of ambiguity of that data take was $36.25 \mathrm{~m}$ and the wavelength was $3.10665 \mathrm{~cm}$. Hence the resulting baseline error in line of sight of this data take is $\Delta B_{\mathrm{LOS}}=4.05 \mathrm{~mm}$. 


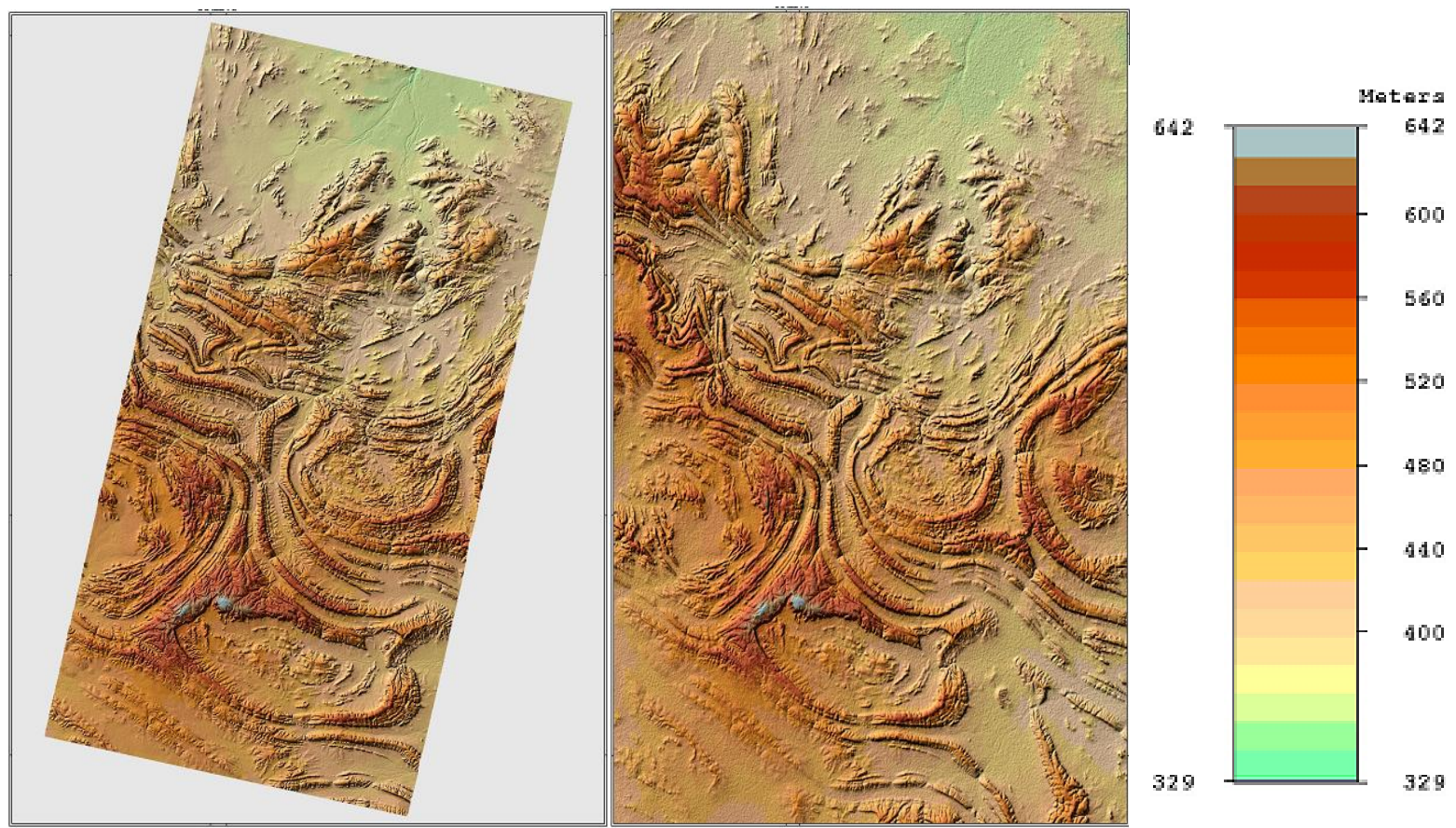

Figure 8. Baseline calibration data take compared to SRTM data

After reduction of outliers, 675 calibration data takes can be used for the estimation of the baseline bias. Fig. 10 shows the detected baseline errors in line of sight in dependence of the incidence angle (each dot represents the result of one baseline calibration data take). 301 data takes were acquired with an incidence angle of $\theta=31^{\circ}$. The baseline error for this group of data takes has a mean value of $0.37 \mathrm{~mm}$ with a standard deviation of $10.10 \mathrm{~mm}$. 70 data takes were acquired with $\theta=40^{\circ}$ with a mean baseline error of $-0.87 \pm 10.84 \mathrm{~mm}$. The remaining 304 data takes were acquired under an incidence angle of $\theta=47^{\circ}$ and show a mean baseline error of $-0.12 \pm 9.42$ $\mathrm{mm}$. The baseline errors vary between $\pm 30 \mathrm{~mm}$ with a standard deviation of about $10 \mathrm{~mm}$. This high dispersion is caused by inaccuracies in the SRTM DEM. 


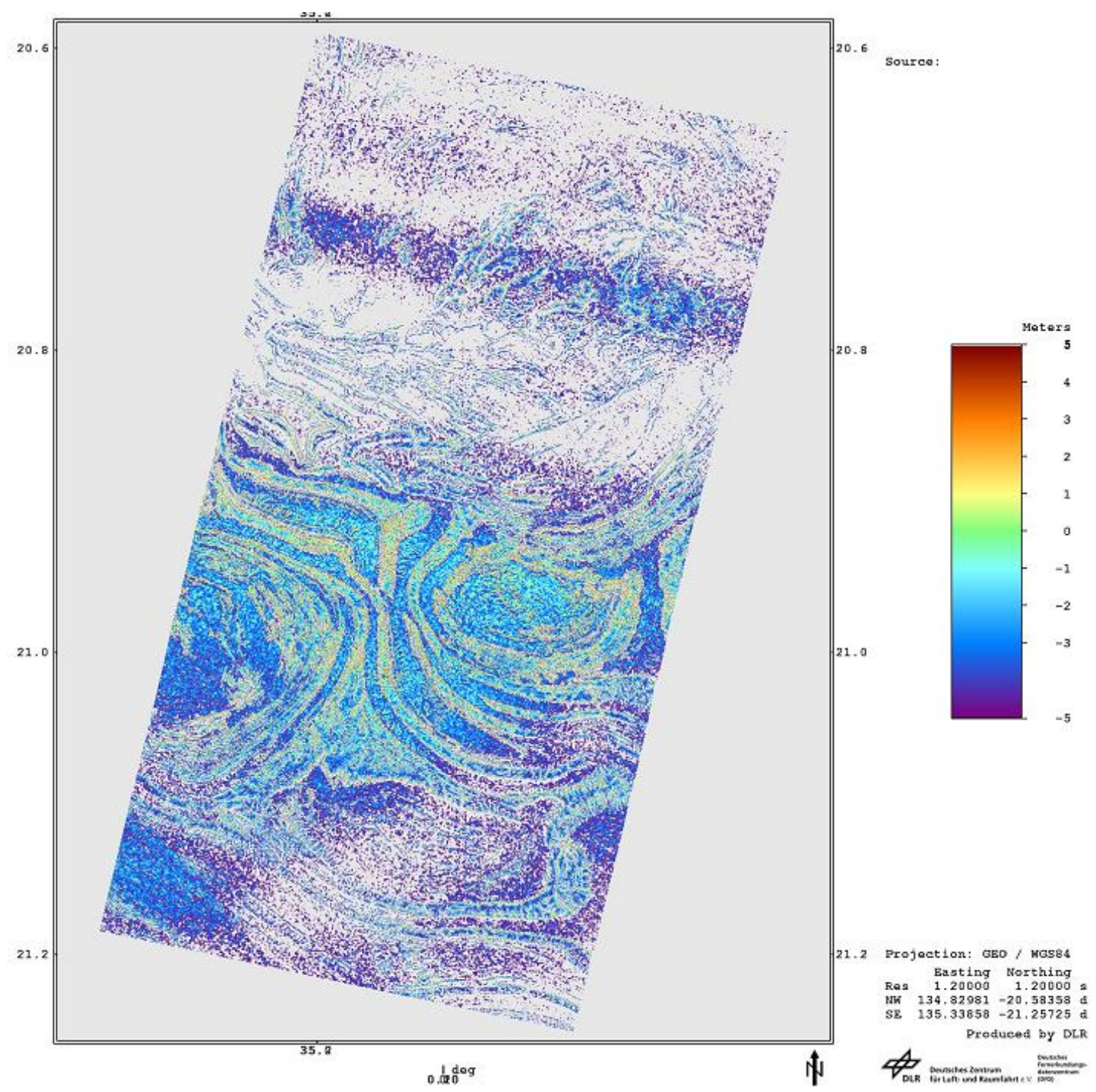

Figure 9. Difference between TDX and SRTM

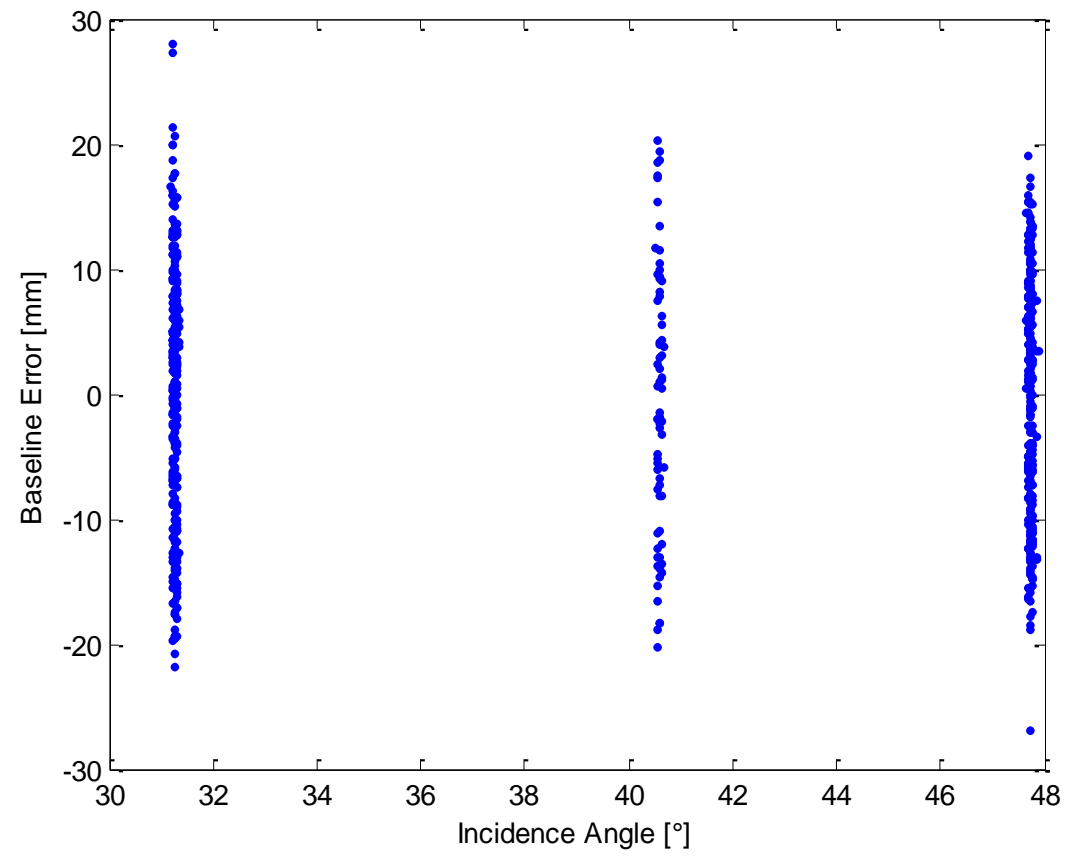

Figure 10. Baseline errors in line of sight 
The baseline bias in radial and normal direction can now be estimated from the baseline errors in line of sight in a least squares adjustment:

$$
\left(\begin{array}{l}
b_{r} \\
b_{n}
\end{array}\right)=\left(\mathbf{A}^{T} \mathbf{A}\right)^{-1} \mathbf{A}^{T} \Delta \mathbf{B}_{L O S},
$$

where $\square \mathbf{B}_{\text {LOS }}$ is a vector containing the baseline errors in line of sight. $\mathbf{A}$ is the design matrix and the $i$-th line corresponds to the observation equation of the $i$-th calibration data take:

$$
\mathbf{A}_{i}=\left(\begin{array}{ll}
-\cos \theta_{i} & -\sin \theta_{i}
\end{array}\right)
$$

This results in a baseline bias in radial direction of $b_{\mathrm{r}}=-1.19 \pm 1.81 \mathrm{~mm}$ and in normal direction of $b_{\mathrm{n}}=1.41 \pm$ $2.17 \mathrm{~mm}$. The size of the determined biases is in the expected order of magnitude. But as the standard deviation of the estimated biases is larger than the biases themselves, the significance of the result is not very high. In addition the two components are highly anti-correlated with a correlation coefficient of -0.96 . This reflects the fact that all data takes are acquired under a similar incidence angle (between $31^{\circ}$ and $47^{\circ}$ degrees). Thus it is difficult to separate the radial and normal component from this geometry.

\section{Conclusions and outlook}

The analysis in this paper has shown that a baseline accuracy similar to that of the GRACE mission can be reached. However, a systematic bias in the expected order of less than $2 \mathrm{~mm}$ per component remains. The bias was estimated by the evaluation of baseline calibration data takes, but the evaluation is not yet highly significant, as the uncertainty of the estimated bias is larger than bias itself. The significance can be increased by adding more baseline calibration data takes to the evaluation and by selecting reference DEMs with a higher accuracy. The radial and normal component of the estimated baseline bias are highly (anti-) correlated due to the data take geometry. This correlation could be resolved by adding left-looking baseline calibration data takes to the evaluation.

Eventually the baseline computed by GSOC will be combined with two baseline solutions computed by GFZ with independent software packages to a so-called calibrated baseline product. This calibrated baseline product will be more accurate than the single baseline solutions. It is expected to fulfill the accuracy requirement of $1 \mathrm{~mm}$ (1DRMS) and will be used for the routine processing of DEM data takes.

\section{Acknowledgements}

The TanDEM-X project is being implemented by a Public-Private Partnership (PPP) between the German Aerospace Center (DLR) and Astrium GmbH.

DLR is responsible for the scientific exploitation of the TanDEM-X data as well as for planning and implementing the mission, controlling the two satellites and generating the digital elevation model. Astrium built the satellite and shares in the cost of its development and exploitation. As with TerraSAR-X, the responsibility for marketing the TanDEM-X data commercially lies in the hands of Infoterra GmbH, a subsidiary of Astrium.

\section{References}

Jäggi, A., Dach, R., Montenbruck, O., Hugentobler, U., Bock, H., Beutler, G., Phase Center Modeling for LEO GPS receiver antennas and its impact on precise orbit determination, Journal of Geodesy 83:1145-1162, DOI 10.1007/s00190-009-0333-2, 2009.

Kahle, R., Schlepp, B., Extending the TerraSAR-X Flight Dynamics System for TanDEM-X, 4th International Conference on Astrodynamics Tools and Techniques, Madrid, 3-6 May 2010.

Krieger, G., Hajnsek, I., Panagiotis, Papathanassiou, K., Younis, M., Moreira, A., Interferometric Synthetic Aperture Radar (SAR) Missions Employing Formation Flying, Proceedings of the IEEE, Vol. 98, No 5, May 2010.

Krieger, G., Moreira, A., Fiedler, H., Hajnsek, I., Werner, M., Younis, M., Zink, M., TanDEM-X: A Satellite Formation for High-Resolution SAR Interferometry, IEEE Transactions on Geoscience and Remote Sensing, Vol. 45, No. 11, November 2007. 
Kroes, R., Precise Relative Positioning of Formation Flying Spacecraft using GPS, PhD Thesis, TU Delft 2006. Montenbruck, O., Wermuth, M., Kahle, R., GPS Based Relative Navigation for the TanDEM-X Mission - First Flight Results, Proceedings ION-GNSS-2010, 22.-24. Sep. Portland OR, USA, 2010. 